\title{
Simplified Machine Diagnosis Techniques under Impact Vibration Using Higher Order Cumulants with the Comparison of Three Cases
}

\author{
Kazuhiro Takeyasu ${ }^{1}$ \\ ${ }^{1}$ College of Business Administration, Tokoha University, Japan \\ Correspondence: Kazuhiro Takeyasu, College of Business Administration, Tokoha University, Japan
}

Received: December 24, 2018

Accepted: January 4, 2019

Online Published: January 11, 2019

doi:10.5539/ibr.v12n2p30

URL: https://doi.org/10.5539/ibr.v12n2p30

\begin{abstract}
Among many amplitude parameters, Kurtosis (4-th normalized moment of probability density function) is recognized to be the sensitive good parameter for machine diagnosis. On the other hand, a new method of machine diagnosis can be considered utilizing the higher order cumulants which have the characteristics that cumulants more than $3^{\text {rd }}$ order are 0 under Gaussian distribution. Cumulants are stated in combination with the same order moment and the moments under that order. Simple calculation method is required on the maintenance site. Furthermore, the absolute deterioration factor such as Bicoherence would be much easier to handle because it takes the value of 1.0 under the normal condition and tends to be 0 when damages increase. In this paper, $\mathrm{n}^{\text {th }}$ normalized cumulant is considered so as to intensify the sensitivity of diagnosis. Also, the simplified calculation method for this new parameter by impact vibration is introduced. Furthermore, the absolute deterioration factor is introduced. Three cases in which the rolling elements number is nine, twelve and sixteen are examined and compared. The new calculation method is examined whether it is a sensitive good parameter or not. Compared with the results obtained so far, the new method shows fairly good results.
\end{abstract}

Keywords: impact vibration, kurtosis, cumulant, rolling element, sensitivity

\section{Introduction}

In mass production firms such as steel making that have big equipments, sudden stops of production processes of machine failure cause great damages such as shortage of materials to the later processes, delays to the due date and the increasing idling time.

To prevent these troubles, machine diagnosis techniques play important roles. So far, Time Based Maintenance (TBM) technique has constituted the main stream of the machine maintenance, which makes checks for maintenance at previously fixed time. But it has a weak point that it makes checks at scheduled time without taking into account whether the parts still keeping good conditions or not. On the other hand, Condition Based Maintenance (CBM) makes maintenance by watching the condition of machines. Therefore, if the parts are still keeping good condition beyond its supposed life, the cost of maintenance may be saved because machines can be used longer than planned. Therefore, the use of CBM has become dominant. The latter one needs less cost of parts, less cost of maintenance and leads to lower failure ratio.

However, it is mandatory to catch a symptom of the failure as soon as possible of a transition from TBM to CBM is to be made. Many methods are developed and examined focusing on this subject. In this paper, we propose a method for the early detection of the failure on rotating machines which is the most common theme in machine failure detection field.

So far, many signal processing methods for machine diagnosis have been proposed (Bolleter, 1998; Hoffner, 1991). As for sensitive parameters, Kurtosis, Bicoherence, Impact Deterioration Factor (ID Factor) were examined (Yamazaki, 1997; Maekawa et al., 1997; Shao et al., 2001; Song et al., 1998; Takeyasu, 1989; Takeyasu et al. 2004; Takeyasu, 2017).

Kurtosis is one of the sophisticated inspection parameter which calculatesnormalized 4th moment of probability density function.

In the industry, there are cases where quick reactions are required on watching the waveform at the machine site.

In this paper, we consider the case such that impact vibration occurs on the gear when the failure arises. Under 
the normal condition, the probability density function of amplitude signal would be the Normal Distribution in general. A new index would be introduced using the relation that higher order cumulants more than 3rd order are 0 under the Normal Distribution. The higher order cumulant is expressed as the combination of the same order moment and the moments less than that order. The new indices would be introduced because they are the combinations of plural order moments. If they are calculated into simple formed equations and their indices are sensitive, the new indices would be expected to be useful.

Furthermore, an absolute deterioration factor such as Bicoherence would be much easier to handle because it takes the value of 1.0 under the normal condition and tends to be 0 when damages increase. Cumulants more than $3^{\text {rd }}$ order are 0 under the normal condition and when failure increases, the value grows big. Therefore, the inverse number of the sum of calculated value of cumulants plus 1 would behave as an absolute index. The new index shows that it is 1.0 under the normal condition and tends to be 0 when failure increases. In this paper, we introduce a simplified calculation method to this new index and name this as a simplified absolute index of higher order cumulants. Three cases in which the rolling elements number is nine, twelve and sixteen are examined and compared.

Trying several orders, we show that the new method is much more sensitive than Bicoherence. This simplified method enables us to calculate the new index even on a pocketsize calculator and enables us to install it in microcomputer chips. We show the fundamental relationship with moments and cumulants in section 2 . We confirm the simplified calculation method of kurtosis we proposed before in section 3 . In section 4 , the simplified absolute index of higher order cumulants are introduced. Numerical examples are exhibited and they are compared with Bicoherence in section 5 which are followed by remarks of section 6 . Section 7 is a summary.

\section{Moment and Cumulant}

\subsection{Moment}

In cyclic movements such as those of bearings and gears, the vibration grows larger whenever the deterioration becomes bigger. Also, it is well known that the vibration grows large when the setting equipment to the ground is unsuitable (Yamazaki, 1977). Assume the vibration signal is the function of time as $\mathrm{x}(\mathrm{t})$. And also assume that it is a stationary time series with mean $\bar{x}$. Denote the probability density function of these time series as $p(x)$. When mean $\bar{x}$ is denoted by

$$
\bar{x}=\int_{-\infty}^{\infty} x p(x) d x
$$

and variance $\sigma^{2}$ is denoted by

$$
\sigma^{2}=\int_{-\infty}^{\infty}(x-\bar{x})^{2} p(x) d x
$$

3rd order moment MT(3), 4th order moment MT(4) are denoted by

$$
\begin{gathered}
M T(3)=\int_{-\infty}^{\infty}(x-\bar{x})^{3} p(x) d x \\
M T(4)=\int_{-\infty}^{\infty}(x-\bar{x})^{4} p(x) d x
\end{gathered}
$$

The normalized index of 3rd order moment and 4th order moment is known as Skewness(SK),Kurtosis(KT) for each in the following definition.

$$
\begin{gathered}
S K=\frac{\int_{-\infty}^{\infty}(x-\bar{x})^{3} p(x) d x}{\left[\int_{-\infty}^{\infty}(x-\bar{x})^{2} p(x) d x\right]^{\frac{3}{2}}} \\
K T=\frac{\int_{-\infty}^{\infty}(x-\bar{x})^{4} p(x) d x}{\left[\int_{-\infty}^{\infty}(x-\bar{x})^{2} p(x) d x\right]^{2}}
\end{gathered}
$$

Discrete time series are stated as follows. 


$$
x_{k}=x(k \Delta t) \quad(k=1,2, \cdots)
$$

where $\Delta t$ is a sampling time interval. Mean $\bar{x}$, variance $\sigma^{2}$, Skewness (SK) and Kurtosis(KT) are stated as follows under discrete time series.

$$
\begin{gathered}
\bar{x}=\lim _{M \rightarrow \infty} \frac{1}{M} \sum_{i=1}^{M} x_{i} \\
\sigma^{2}=\lim _{M \rightarrow \infty} \frac{1}{M} \sum_{i=1}^{M}\left(x_{i}-\bar{x}\right)^{2} \\
M T(3)=\lim _{M \rightarrow \infty} \frac{1}{M} \sum_{i=1}^{M}\left(x_{i}-\bar{x}\right)^{3} \\
M T(4)=\lim _{M \rightarrow \infty} \frac{1}{M} \sum_{i=1}^{M}\left(x_{i}-\bar{x}\right)^{4} \\
S K=\lim _{M \rightarrow \infty} \frac{\frac{1}{M} \sum_{i=1}^{M}\left(x_{i}-\bar{x}\right)^{3}}{\left\{\frac{1}{M} \sum_{i=1}^{M}\left(x_{i}-\bar{x}\right)^{2}\right\}^{\frac{3}{2}}} \\
K T=\lim _{M \rightarrow \infty} \frac{1}{\left\{\frac{1}{M} \sum_{i=1}^{M}\left(x_{i}-\bar{x}\right)^{4}\left(x_{i}-\bar{x}\right)^{2}\right\}^{2}}
\end{gathered}
$$

\subsection{Cumulant}

Characteristic function $\varphi(u)$ is defined as follows (Hino, 1977).

$$
\varphi(u)=\int_{-\infty}^{\infty} e^{i u x} p(x) d x
$$

After taking Taylor expansion of the characteristic function, we can obtain coefficient $c(n)$ as:

$$
\begin{aligned}
& \ln \varphi(u)=\sum_{n=1}^{\infty} c(n) \frac{i^{n}}{n !} u^{n} \\
& c(n)=\left[\frac{(-i)^{n} d^{n} \ln \varphi(u)}{d u^{n}}\right]_{u=0}
\end{aligned}
$$

This $c(n)$ is called cumulant. There exists following relation between cumulant and moment.

$$
\begin{aligned}
& M T(n)=\sum_{\lambda^{(1)}+\cdots+\lambda^{(q)}=v} \frac{1}{q !} \frac{v !}{\lambda^{(1)} ! \cdots \lambda^{(q)} !} \prod_{n=1}^{q} c(n) \\
& c(n)=\sum_{\lambda^{(1)}+\cdots+\lambda^{(q)}=v} \frac{(-1)^{q-1}}{q !} \frac{v !}{\lambda^{(1)} ! \cdots \lambda^{(q)} !} \prod_{n=1}^{q} M T(n)
\end{aligned}
$$


When $\bar{x}=0$, Eq.(17) becomes as follows for $\mathrm{n}=1,2,3,4,6,8$.

$$
\begin{gathered}
c(1)=\bar{x}=0 \\
c(2)=\sigma^{2} \\
c(3)=M T(3) \\
c(4)=M T(4)-3\{M T(2)\}^{2} \\
c(6)=M T(6)-15 M T(4) M T(2)-10\{M T(3)\}^{2}+30\{M T(2)\}^{3} \\
c(8)=M T(8)-28 M T(6) M T(2)-56 M T(5) M T(3)-35\{M T(4)\}^{2}+420 M T(4)\{M T(2)\}^{2} \\
+560\{M T(3)\}^{2} M T(2)-630\{M T(2)\}^{4}
\end{gathered}
$$

Where MT(6),MT(8) are $6^{\text {th }}$ moment, $8^{\text {th }}$ moment for each. It is well known that cumulant is 0 for the higher order cumulant more than 3 when the probability density function is a normal distribution.

\subsection{Bicoherence}

Bicoherence shows the relationship between two frequencies and is expressed as:

$$
\text { Bic },_{x x x}\left(f_{1}, f_{2}\right)=\frac{B_{x x x}\left(f_{1}, f_{2}\right)}{\sqrt{S_{x x}\left(f_{1}\right) \cdot S_{x x}\left(f_{2}\right) \cdot S_{x x}\left(f_{1}+f_{2}\right)}}
$$

Here

$$
B_{x x x}\left(f_{1}, f_{2}\right)=\frac{X_{T}\left(f_{1}\right) \cdot X_{T}\left(f_{2}\right) \cdot X^{*}{ }_{T}\left(f_{1}+f_{2}\right)}{T^{\frac{3}{2}}}
$$

means Bispectrum and

$$
X_{T}(t)= \begin{cases}x(t) & (0<t<T) \\ 0 & (e l) e\end{cases}
$$

T: Basic Frequency Interval

$$
\begin{array}{r}
X_{T}(f)=\int_{-\infty}^{\infty} X_{T}(t) e^{-j 2 \pi f t} d t \\
S_{x x}(f)=\frac{1}{T} X_{T}(f) X^{*}{ }_{T}(f)
\end{array}
$$

Range of Bicoherence satisfies:

$$
0<\operatorname{Bic},_{x x x}\left(f_{1}, f_{2}\right)<1
$$

\section{Simplified Calculation Method of Kurtosis}

Assume that we get $N$ amount of data and then newly get $l$ amount of data. Let mean, variance, $M T(3), M T(4), S K, K T$ of $1 \sim N$ data state as $\bar{x}_{N} 、 \sigma^{2}{ }_{N} 、 M T_{N}(3) 、 M T_{N}(4) 、 S K_{N} 、 K T_{N}$ 
for each. And as for $N+1 \sim N+l$ data, we state above items as

$$
\bar{x}_{N / l}, \sigma_{N / l}^{2}, M T_{N / l}(3) 、 M T_{N / l}(4) 、 S K_{N / l} 、 K T_{N / l}
$$

For example, $M T_{N}(4)$ and $M T_{N / l}(4)$ are stated as follows.

$$
\begin{aligned}
& M T_{N}(4)=\frac{1}{N} \sum_{i=1}^{N}\left(x_{i}-\bar{x}\right)^{4} \\
& M T_{N / l}(4)=\frac{1}{l} \sum_{i=N+1}^{N+l}\left(x_{i}-\bar{x}\right)^{4}
\end{aligned}
$$

When there arise failures on bearings or gears, peak values arise cyclically. In the early stage of the defect, this peak signal usually appears clearly. Generally, defects will injure other bearings or gears by contacting the covering surface as time passes. Assume that the peak signals of $S$ times magnitude from the normal signals arise during $m$ times measurement of samplings. As for determining sampling interval, the sampling theorem is well known (Tokumaru et al., 1982). But in this paper, we do not pay much attention on this point in order to focus on our proposal theme. Let $\sigma^{2}{ }_{N / l}$ and $M T_{N / l}(4)$ of this case denote $\bar{\sigma}_{N / l}^{2}, \overline{M T}_{N / l}(4)$, then we get

$$
\begin{gathered}
\bar{\sigma}_{N / l}^{2}=\frac{1}{l} \sum_{i=N+1}^{N+l}\left(x_{i}-\bar{x}\right)^{2} \\
\cong \frac{l-\frac{l}{m}}{l} \sigma^{2}+\frac{\frac{l}{m}}{l} S^{2} \sigma^{2}{ }_{N} \\
=\sigma^{2}{ }_{N}\left(1+\frac{S^{2}-1}{m}\right) \\
\frac{l}{M T}{ }_{N / l}(4)=\frac{1}{l} \sum_{i=N+1}^{N+l}\left(x_{i}-\bar{x}\right)^{4} \\
\cong \frac{l}{l} M T_{N / l}(4)+\frac{\frac{l}{l}}{l} S^{4} M T_{N / l}(4) \\
=\left(1+\frac{S^{4}-1}{m}\right) M T_{N / l}(4)
\end{gathered}
$$

We assume that time series are stationary as is stated at 2.1. Therefore, even if sample pass may differ, mean and variance is naturally supposed to be same when the signal is obtained from the same data occurrence point of the same machine.

We consider such case that the impact vibration occurs. Except for the impact vibration, other signals are assumed to be stationary and have the same means and variances. Under this assumption, we can derive the simplified calculation method for machine diagnosis which is a very practical one.

For these equations, we obtain $\overline{K T}_{N+l}$ as $K T_{N+l}$ of the above case 


$$
\begin{aligned}
& \overline{K T}_{N+l} \cong \frac{\frac{N}{N+l} M T_{N}(4)+\frac{l}{N+l}\left(1+\frac{S^{4}-1}{m}\right) M T_{N}(4)}{\left\{\frac{N}{N+l} \sigma^{2}{ }_{N}+\frac{l}{N+l} \sigma_{N}^{2}\left(1+\frac{S^{2}-1}{m}\right)\right\}^{2}} \\
&=K T_{N} \frac{\left(1+\frac{l}{N+l} \frac{S^{4}-1}{m}\right)}{\left(1+\frac{l}{N+l} \frac{S^{2}-1}{m}\right)^{2}}
\end{aligned}
$$

While $K T_{N}=3.0$ under normal condition.

n.b.

Under the following Normal distribution,

$$
\phi(x)=\frac{1}{\sqrt{2 \pi} \sigma} e^{-\frac{1}{2}\left(\frac{x-\bar{x}}{\sigma}\right)^{2}}
$$

Its moment is described as follows which is well known (Hino, 1977).

$$
\begin{aligned}
& \operatorname{MT}(2 n-1)=0 \\
& \operatorname{MT}(2 n)=\prod_{k=1}^{n}(2 k-1) \sigma^{2 n}
\end{aligned}
$$

\section{Simplified Absolute Index of the Higher Order Cumulant}

\subsection{Absolute Index of Higher Order Cumulant}

Under the normal distribution, the higher order cumulants more than $3^{\text {rd }}$ order are 0 . If the system is under the normal condition, we may suppose $p(x)$ becomes a normal distribution function. The normalized cumulant $\mathrm{CT}$ is as follows which is the same form of Skewness and Kurtosis at 3.

As

$$
\begin{aligned}
K T_{N} & =\frac{M T_{N}(4)}{\sigma_{N}^{4}} \\
\overline{K T}_{N+l} & =\frac{\overline{M T}_{N+l}(4)}{\bar{\sigma}_{N+l}^{4}}
\end{aligned}
$$

in the same way, we obtain

$$
\begin{gathered}
C T_{N}(n)=\frac{C_{N}(n)}{\sigma_{N}{ }^{n}} \\
\overline{C T}_{N+l}(n)=\sum_{\lambda^{(1)}+\cdots+\lambda^{(q)}=v} \frac{(-1)^{q-1}}{q !} \frac{v !}{\lambda^{(1)} ! \cdots \lambda^{(q)} !} \prod_{n=1}^{q} \overline{M T}_{N+l}(n) \frac{1}{\bar{\sigma}_{N+l}^{n}}
\end{gathered}
$$




$$
\cong \sum_{\lambda^{(1)}+\cdots+\lambda^{(q)}=v} \frac{(-1)^{q-1}}{q !} \frac{v !}{\lambda^{(1)} ! \cdots \lambda^{(q)} !} \prod_{n=1}^{q} \frac{1+\frac{l}{N+l} \frac{S^{n}-1}{m}}{\left(1+\frac{l}{N+l} \frac{S^{2}-1}{m}\right)^{\frac{n}{2}}} \prod_{i=1}^{\frac{n}{2}}(2 i-1)
$$

Therefore, the simplified absolute index of the higher order cumulant is described as follows.

$$
\begin{gathered}
\overline{Z c}_{N+l}(n)=\frac{1}{1+\bar{c}_{N+l}(n)} \\
=\frac{1}{1+\sum_{\lambda^{(1)}+\cdots+\lambda^{(q)}=v} \frac{(-1)^{q-1}}{q !} \frac{v !}{\lambda^{(1)} ! \cdots \lambda^{(q)} !} \prod_{n=1}^{q} \overline{M T}(n)} \\
\cong \frac{1}{1+\sum_{\lambda^{(1)}+\cdots+\lambda^{(q)}=v} \frac{(-1)^{q-1}}{q !} \frac{v !}{\lambda^{(1)} ! \cdots \lambda^{(q)} !} \prod_{n=1}^{q} \frac{1+\frac{l}{N+l} \frac{S^{n}-1}{m}}{\left(1+\frac{l}{N+l} \frac{S^{2}-1}{m}\right)^{\frac{n}{2}}} \prod_{i=1}^{\frac{n}{2}}(2 i-1) \sigma^{n}}
\end{gathered}
$$

This is the absolute deterioration factor of which range is 1 to 0 .

\subsection{Simplified Calculation Method for the Higher Order Cumulant}

3rd order cumulant is 3rd order moment itself as is expressed in Eq.(20). Therefore, we can obtain the following equations. When the peak signals arise, we denote $c_{N+l}(3)$ to be $\bar{c}_{N+l}(3)$ as before for $N+l$ data.

$$
\begin{gathered}
\bar{c}_{N+l}(3)=\overline{M T}_{N+l}(3) \\
\cong \frac{1}{N+l} M T_{N}(3)+\frac{l}{N+l}\left(1+\frac{S^{3}-1}{m}\right) M T_{N}(3) \\
=\left(1+\frac{l}{N+l} \frac{S^{3}-1}{m}\right) M T_{N}(3)
\end{gathered}
$$

Under the normal condition or the probability density function has the symmetric form for right and left even if impact vibration occurs, $M T_{N}(3)$ is 0 . Therefore $\bar{c}_{N+l}(3)$ is also 0 . We can obtain the following 4 th cumulant from Eq.(21).

$$
\begin{aligned}
& \bar{c}_{n+l}(4)=\overline{M T}_{N+l}(4)-3 \overline{M T}_{N+l}(2)^{2} \\
& \cong\left(1+\frac{l}{N+l} \frac{S^{4}-1}{m}\right) M T_{N}(4)-3\left(1+\frac{l}{N+l} \frac{S^{2}-1}{m}\right)^{2} \sigma^{4}{ }_{N}
\end{aligned}
$$

Under the normal condition, the following equation is derived because $M T_{N}(4) \cong 3 \sigma^{4}{ }_{N}$ by Eq.(35).

$$
\bar{c}_{N+l}(4) \cong 3 \cdot \frac{l}{N+l}\left(1-\frac{l}{N+l} \cdot \frac{1}{m}\right) \frac{(S+1)^{2}(S-1)^{2}}{m} \sigma^{4}{ }_{N}
$$


Under the normal condition, data can be normalized to be $\bar{x}=0, \sigma^{2}=1$ without loss of generality (Tokumaru et al., 1982). Hence, we calculate using the following equation hereafter.

$$
\bar{c}_{N+l}(4) \cong 3 \cdot \frac{l}{N+l}\left(1-\frac{l}{N+l} \cdot \frac{1}{m}\right) \frac{(S+1)^{2}(S-1)^{2}}{m}
$$

In the same way, 6th cumulant and 8th cumulant are described as follows from Eq(22),(23).

$$
\begin{array}{r}
\bar{c}_{N+l}(6) \cong 15 \cdot \frac{l}{N+l} \frac{1}{m}\left\{1+\frac{l}{N+l} \cdot \frac{1}{m}\left(-3+2 \cdot \frac{l}{N+l} \frac{1}{m}\right)\right\}\left(S^{2}-1\right)^{3} \\
\bar{c}_{N+l}(8) \cong 105 \cdot \frac{l}{N+l} \frac{1}{m}\left[1-\frac{l}{N+l} \frac{1}{m}\left\{7-6 \cdot \frac{l}{N+l} \frac{1}{m}\left(2-\frac{l}{N+l} \frac{1}{m}\right)\right\}\right]\left(S^{2}-1\right)^{4}
\end{array}
$$

\section{Numerical Examples}

If the system is under the normal condition, we may suppose $p(x)$ becomes a normal distribution function. Under the assumption of 3 , let $m=9,12,16$, considering the cases $S=2,3, \cdots, 6$ for 3 ., and setting $l=\frac{N}{10}$, we obtain Table 1 from the calculation of $\overline{Z c}_{N+l}(n)$. Here, $m$ is the number of rolling elements.

Table 1. Transition of $\overline{Z c}_{N+l}(n) \quad\left(l=\frac{N}{10}\right)$

$<\mathrm{m}=9>$

\begin{tabular}{lllllll}
\hline & $\mathbf{S}=\mathbf{1}$ & $\mathbf{2}$ & $\mathbf{3}$ & $\mathbf{4}$ & $\mathbf{5}$ & $\mathbf{6}$ \\
\hline $\mathrm{n}=4$ & 1 & 0.78742 & 0.34249 & 0.12904 & 0.05471 & 0.02649 \\
$\mathrm{n}=6$ & 1 & 0.20130 & 0.01312 & 0.00201 & 0.00049 & 0.00016 \\
\hline
\end{tabular}

$<\mathrm{m}=12>$

\begin{tabular}{lllllll}
\hline & $\mathbf{S}=\mathbf{1}$ & $\mathbf{2}$ & $\mathbf{3}$ & $\mathbf{4}$ & $\mathbf{5}$ & $\mathbf{6}$ \\
\hline $\mathrm{n}=4$ & 1 & 0.83126 & 0.40925 & 0.16461 & 0.07147 & 0.03493 \\
$\mathrm{n}=6$ & 1 & 0.25007 & 0.01728 & 0.00266 & 0.00065 & 0.00021 \\
\hline
\end{tabular}

\begin{tabular}{cllllll}
$\langle\mathrm{m}=16>$ & \multicolumn{5}{l}{} \\
\hline & $\mathbf{S}=\mathbf{1}$ & $\mathbf{2}$ & $\mathbf{3}$ & $\mathbf{4}$ & $\mathbf{5}$ & $\mathbf{6}$ \\
\hline $\mathrm{n}=4$ & 1 & 0.86765 & 0.47968 & 0.20775 & 0.09292 & 0.04595 \\
$\mathrm{n}=6$ & 1 & 0.30657 & 0.02278 & 0.00352 & 0.00086 & 0.00028 \\
\hline
\end{tabular}

Next, setting $N \rightarrow 0, l \rightarrow N$, we obtain Table 2 .

Table 2. Transition of $\overline{Z c}_{N+l}(n) \quad(N=\varepsilon(\varepsilon \rightarrow 0), l=N)$

$<\mathrm{m}=9>$

\begin{tabular}{lllllll}
\hline & $\mathbf{S}=\mathbf{1}$ & $\mathbf{2}$ & $\mathbf{3}$ & $\mathbf{4}$ & $\mathbf{5}$ & $\mathbf{6}$ \\
\hline $\mathrm{n}=4$ & 1 & 0.27273 & 0.05009 & 0.01478 & 0.00583 & 0.00275 \\
$\mathrm{n}=6$ & 1 & 0.03114 & 0.00169 & 0.00026 & 0.00006 & 0.00002 \\
\hline
\end{tabular}

\begin{tabular}{cllllll}
$\langle\mathrm{m}=12>$ & \multicolumn{1}{l}{} \\
\hline & $\mathbf{S}=\mathbf{1}$ & $\mathbf{2}$ & $\mathbf{3}$ & $\mathbf{4}$ & $\mathbf{5}$ & $\mathbf{6}$ \\
\hline $\mathrm{n}=4$ & 1 & 0.32653 & 0.06383 & 0.01903 & 0.00752 & 0.00355 \\
$\mathrm{n}=6$ & 1 & 0.03767 & 0.00204 & 0.00031 & 0.00008 & 0.00002 \\
\hline
\end{tabular}




\begin{tabular}{cllllll}
$\langle\mathrm{m}=16>$ & \multicolumn{1}{l}{} \\
\hline & $\mathbf{S = 1}$ & $\mathbf{2}$ & $\mathbf{3}$ & $\mathbf{4}$ & $\mathbf{5}$ & $\mathbf{6}$ \\
\hline $\mathrm{n}=4$ & 1 & 0.38729 & 0.08163 & 0.02466 & 0.00998 & 0.00462 \\
$\mathrm{n}=6$ & 1 & 0.04595 & 0.00253 & 0.00039 & 0.00009 & 0.00003 \\
\hline
\end{tabular}

As the value at $n=6$ is already so small, we have skipped the calculation at $n=8$. It does not affect the consideration of the result.

As $\mathrm{S}$ grows large, the value decreases rapidly. As $m$ increases, the value increases slightly.

As for higher order cumuulants cases such as $n=6$, sensitivity grow much better. But calculation becomes more complicated as $n$ grows large.

Each corresponding case of Table 2 shows much more proceeded value in deterioration than those of Table 1. It is because each case of Table 2 is occupied only by the data under irregular condition.

Subsequently, we examine Bicoherence. We made experiment in the past (Takeyasu,1987,1989).

Summary of the experiment is as follows. Pitching defects are pressed on the gears of small testing machine.

Small defect condition: Pitching defects pressed on $1 / 3$ gears of the total gear.

Middle defect condition: Pitching defects pressed on 2/3 gears of the total gear.

Big defect condition: Pitching defects pressed on whole gears of the total gear.

We examined several cases for the $f_{1}, f_{2}$ in Eq.(24). We got the best-fit result in the following case.

$$
\left\{\begin{array}{l}
f_{1}: \text { peak frequency of power spectrum } \\
f_{2}: 2 f_{1}
\end{array}\right.
$$

We obtained the following Bicoherence values in this case (Table 3).

Table 3. Transition of Bicoherence value

\begin{tabular}{cc}
\hline Condition & Bicoherence \\
\hline Normal & 0.99 \\
Small defect & 0.38 \\
Middle defect & 0.09 \\
Big defect & 0.02 \\
\hline
\end{tabular}

Thus, Bicoherence proved to be a very sensitive good index. Bicoherence is an absolute index of which range is 1 to 0 .

Therefore it can be said that it is a universal index.

In those experiment, small defect condition is generally assumed to be $S=2$ and big defect condition is generally assumed to be $S=6$ (Maekawa,K. et al. 1997). Therefore, approximate comparison may be achieved, though the condition does not necessarily coincide.

In the case of $n=4$ in Table 2, the value is 0.273 at small defect condition and 0.015 at middle defect condition and 0.003 at big defect condition which show more sensitive behavior than Bicoherence. In the case of $n=6$, the value decreases so heavily. Similar things can be said for $\mathrm{m}=12$ and $\mathrm{m}=16$. It could be said that the case $n=4$ would be sensitive enough for the practical use. Therefore, the case $n=4$ would be recommended in this new method. This calculation method is simple enough to be executed even on a pocketsize calculator. Compared with Bicoherence which has to be calculated by Eq.(24) (27), the proposed method is by far a simple one and easy to handle on the field defection.

\section{Remarks}

The steps for the failure detection by this method are as follows.

1. Prepare standard $\overline{Z c}$ Table for each normal or abnormal level

2. Measure peak values by signal data and compare the peak ratio $(S)$ to the normal data

3. Calculate $\overline{Z c}$ 
4. Judge the failure level by the score of $\overline{Z c}$

$m$ is the value of each equipment. For example, the number of ball bearings or the number of gears. Preparing the standard Table of $\overline{Z c}$ for each normal and abnormal level, we can easily judge the failure level only by taking ratio of the peak value to the normal level and calculating $\overline{Z c}$. This calculation method is very simple and is very practical at the factory of maintenance site. This can be installed in microcomputer chips and utilized as the tool for early stage detection of the failure.

\section{Conclusion}

We proposed a simplified calculation method for an absolute index of higher order cumulant and named this as simplified absolute index of higher order cumulant. Three cases in which the rolling elements number was nine, twelve and sixteen were examined and compared. As $\mathrm{S}$ increases, the value rapidly decreases. As $\mathrm{m}$ increases, the value increases slightly. Compared with the results obtained so far, the results of numerical examples of this paper are reasonable and much more sensitive than another method such as Bicoherence. Judging from these results, our method is properly considered to be effective for the early stage failure detection especially. Although it has a limitation that it is restricted in the number of research, we could obtain the fruitful results. To confirm the findings by utilizing the new consecutive visiting records would be the future works to be investigated.

The effectiveness of this method should be examined in various cases.

\section{References}

Bolleter, U. (1988). Blade Passage Tones of Centrifugal Pumps. Vibration, 4(3), 8-13.

Hino, M. (1977). Spectrum Analysis (in Japanese). Asakura Shoten Publishing.

Hoffner, J. (1991). Preventive maintenance for No-Twist rod mills using vibration signature analysis. Iron and Steel Engineer, 68(1), 55-61.

Maekawa, K., Nakajima, S., \& Toyoda, T. (1997). New Severity Index for Failures of Machine Elements by Impact Vibration (In Japanese). J.SOPE Japan, 9(3), 163-168.

Shao, Y., Nezu, K., Matsuura, T., Hasegawa, Y., \& Kansawa, N. (2001). Bearing Fault Diagnosis Using an Adaptive Filter (in Japanese). J.SOPE Japan, 12(3), 71-77.

Song, J. W., Tin, H., \& Toyoda, T. (1998). Diagnosis Method for a Gear Equipment by Sequential Fuggy Neural Network (in Japanese). J.SOPE Japan, 10(1), 15-20.

Takeyasu, K. (1987). Watching Method of Circulating Moving Object (in Japanese). Certified Patent by Japanese Patent Agency.

Takeyasu, K. (1989). Watching Method of Circulating Moving Object (in Japanese). Certified Patent by Japanese Patent Agency.

Takeyasu, K. (2017). Simplified Machine Diagnosis Techniques by Impact Vibration using 4-th Moment of Absolute Deterioration Factor. Journal of Computations \& Modelling, 7(2), 69-82.

Takeyasu, K., Amemiya, T., Tanaka, J., \& Masuda, S. (2004).Simplified Machine Diagnosis Techniques by Impact Vibration Using n-th Moment of Absolute Deterioration Factor, The 33rd International Conference on Computers and Industrial Engineering.

Tokumaru, H., Soeda, T., Nakamizo, T., \& Akizuki, K. (1982). Measurement and Calcula-tion (in Japanese). Baifukan Publishing.

Yamazaki, H. (1977). Failure Detection and Prediction (In Japanese). Kogyo Chosakai Publishing.

\section{Copyrights}

Copyright for this article is retained by the author(s), with first publication rights granted to the journal.

This is an open-access article distributed under the terms and conditions of the Creative Commons Attribution license (http://creativecommons.org/licenses/by/4.0/). 